\title{
Adaptive beamforming with multiple-beam constraints in the presence of coherent jammers
}

\author{
Ju-Hong Lee*, Ti-Fei Hsu \\ Room 517, Building 2, Department of Electrical Engineering, National Taiwan University, Taipei, 106 Taiwan, ROC
}

Received 28 June 1999; received in revised form 27 March 2000

\begin{abstract}
This paper deals with the problem of adaptive beamforming using a uniform linear array to provide multiple-beam constraints and suppress coherent jammers simultaneously. By constructing a steering matrix with each column vector corresponding to the steering vector of a selective beam and a constraint vector with each entry equal to the gain of a selective beam, we can achieve an adaptive array beamformer with multiple-beam constraints. For coping with the performance degradation due to the presence of coherent jammers, a matrix reconstruction scheme in conjunction with an iterative algorithm is presented to alleviate the coherent jamming effect. Simulation examples for illustration and comparison are also given. (C) 2000 Elsevier Science B.V. All rights reserved.
\end{abstract}

\section{Zusammenfassung}

Die Arbeit beschäftigt sich mit dem Problem der adaptiven Strahlerzeugung durch eine regelmäßig angeordnete Antennenanordnung, um gleichzeitig Randbedingungen für mehrere Strahlrichtungen zu ermöglichen und kohärente Störer zu unterdrücken. Durch die Konstruktion einer Steuermatrix, bei der jedem Spaltenvektor ein Steuervektor eines selektiven Strahls entspricht, und einem Bedingungsvektor, dessen Komponenten den Verstärkungen eines selektiven Strahls entsprechen, können wir eine adaptive Strahlerzeugung mit Bedingungen für Mehrfachstrahlen erreichen. Die mögliche Leistungs-beeinträchtigung durch die Anwesenheit kohärenter Störer wird durch ein Verfahren der Matrixumformung umgegangen, wobei ein iterativer Algorithmus vorgestellt wird, der den Effekt kohärenter Störer vermeidet. Es werden Simulationsbeispiele zur Erläuterung und für Vergleiche angegeben. (C) 2000 Elsevier Science B.V. All rights reserved.

\section{Résumé}

Cer article traite du problème du formatage de voie adaptatif sur un réseau linéaire uniforme dans l'optique de simultanément fournir des contraintes sur des faiscaux multiples et de supprimer des brouilleurs cohérents. Par construction d'une matrice directionnelle dont chaque colonne correspond au vecteur directionnel d'un faisceau sélectif et d'un vecteur de contrainte dont chaque composante est égale au gain d'un faisceau sélectif, nous pouvons obtenir un formateur de réseau adaptatif avec des contraintes de faisceaux multiples. Pour combattre la dégradation de performances due à la présence de brouilleurs cohérents, nous présentons une technique de reconstruction de matrice en conjonction avec un algorithme itératif pour diminuer l'effet du brouillage cohérent. Des exemples de simulation à des fins d'illustration et de comparaison sont également donnés. (C) 2000 Elsevier Science B.V. All rights reserved.

Keywords: Beamforming; Adaptive array; Multiple beams

\footnotetext{
This work was supported by the National Science Council under Grant NSC88-2218-E002-027.

* Corresponding author.
} 


\section{Introduction}

In many applications, such as satellite communications [4], an antenna array must possess beamforming capability to receive more than one signal with specified gain requirements while suppressing all jammers. This purpose can be effectively achieved by using an antenna array with multiplebeam pattern $[4,8]$. In [8], an adaptive algorithm was proposed to find an adaptive weight close to a desired quiescent beam pattern under a unit norm constraint on the weight. However, the resulting problem to be solved is a non-linear optimization problem and, hence, solving it requires a sophisticated procedure as shown in [8]. Moreover, the existing methods for synthesizing an antenna array with multiple-beam pattern cannot deal with the situation where there exists coherence between a desired signal and the jammers.

In this paper, we present a technique for adaptive beamforming with capability of providing multiple-beam constraints (MBC) in addition to coherent jamming suppression. To satisfy the first goal, we formulate the problem as finding such an array weight vector that the array output power is minimized subject to MBC. It is shown that an analytical solution for the resulting optimization problem can be easily obtained. To achieve the second goal, a matrix reconstruction scheme in conjunction with an iterative algorithm is presented to cure the array performance deterioration due to coherent jammers. Simulation results demonstrate the effectiveness of the proposed technique.

\section{Adaptive beamforming with multiple-beam constraints}

In this section, we formulate the problem for adaptive beamforming with capability of receiving more than one desired signals. Consider a uniform linear array (ULA) with $M$ sensors and interelement spacing equal to $\lambda / 2$, where $\lambda$ is the smallest signal wavelength of the signals with specified gain/null arrangements. Assume that $K$ narrowband and far-field signals are impinging on the array from direction angles $\theta_{i}, i=1,2, \ldots, K$ off broadside. The signal received at the $m$ th array sensor can be expressed as

$$
x_{m}(t)=\sum_{i=1}^{K} s_{i}(t) a_{m}\left(\theta_{i}\right)+n_{m}(t), \quad m=1,2, \ldots, M,
$$

where $a_{m}\left(\theta_{i}\right)=\exp \left(j\left(2 \pi d_{m} \sin \theta_{i}\right) / \lambda\right)$ and $d_{m}$ is the distance between the $m$ th and the first array sensors, $s_{i}(t)$ is the complex waveform of the $i$ th signal, and $n_{m}(t)$ is the spatially white noise with mean zero and variance $\sigma_{n}^{2}$ received at the $m$ th array sensor. The corresponding data vector received by the array can be written as

$\boldsymbol{x}(t)=\boldsymbol{A s}(t)+\boldsymbol{n}(t)$,

where $\boldsymbol{A}=\left[a\left(\theta_{1}\right) a\left(\theta_{2}\right) \ldots a\left(\theta_{K}\right)\right]$ with the direction vector of the $i$ th signal given by $a\left(\theta_{i}\right)=$ $\left[a_{1}\left(\theta_{i}\right) a_{2}\left(\theta_{i}\right) \ldots a_{M}\left(\theta_{i}\right)\right]^{\mathrm{T}}$, the signal source vector is $\boldsymbol{s}(t)=\left[s_{1}(t) s_{2}(t) \ldots s_{K}(t)\right]^{\mathrm{T}}$, and the noise vector is $\boldsymbol{n}(t)=\left[n_{1}(t) n_{2}(t) \ldots n_{M}(t)\right]^{\mathrm{T}}$. The superscript T denotes transpose operation. Assume that $\boldsymbol{s}(t)$ and $\boldsymbol{n}(t)$ are uncorrelated. Then the ensemble correlation matrix of $\boldsymbol{x}(t)$ is Toeplitz-Hermitian with size $M \times M$ and given by

$$
\begin{aligned}
\boldsymbol{R}_{x} & =\left[R_{i j}\right]=[R(i-j)]=E\left\{\boldsymbol{x}(t) \boldsymbol{x}^{\mathrm{H}}(t)\right\} \\
& =\boldsymbol{A} \boldsymbol{S} \boldsymbol{A}^{\mathrm{H}}+\sigma_{n}^{2} \boldsymbol{I},
\end{aligned}
$$

where the superscript $\mathrm{H}$ denotes the complex conjugate transpose. $\boldsymbol{S}=E\left\{\boldsymbol{s}(t) \mathbf{s}^{\mathrm{H}}(t)\right\}$ has rank $(K-J+1)$, where $J$ denotes the number of coherent signals.

Consider the application in a communication system where more than one signal reception is required. Based on the Frost's algorithm [2], we can utilize an adaptive antenna array which possesses the capability to provide selective gain/null arrangements for different signal beams while suppressing all jammers. Let the ULA uses a weight vector $\boldsymbol{w}=\left[\begin{array}{llll}w_{1} & w_{2} & \ldots & w_{M}\end{array}\right]^{\mathrm{T}}$ for processing the received data vector $\boldsymbol{x}(t)$. Then the signal at the array output is given by $y(t)=\boldsymbol{w}^{\mathrm{H}} \boldsymbol{x}(t)$. Assume that the selective gain/null requirements are specified by assigning a gain $c_{j}$ at the direction vector $a\left(\theta_{j}\right)$ for $j=1,2, \ldots P$, where $P$ denotes the number of signals with gain/null constraint. Consequently, the problem can be formulated by the following constrained optimization problem:

$$
\begin{array}{ll}
\text { minimize } & E\left\{|y(t)|^{2}\right\}=\boldsymbol{w}^{\mathrm{H}} \boldsymbol{R}_{x} \boldsymbol{w} \\
\text { subject to } & \boldsymbol{G}^{\mathrm{H}} \boldsymbol{w}=\boldsymbol{c},
\end{array}
$$


where $\boldsymbol{G}=\left[a\left(\theta_{1}\right) a\left(\theta_{2}\right) \ldots a\left(\theta_{P}\right)\right]$ denotes the constraint matrix and $c=\left[\begin{array}{llll}c_{1} & c_{2} & \ldots & c_{P}\end{array}\right]^{\mathrm{T}}$ the corresponding gain vector. According to the theory of Compton [1], the optimal solution, i.e., the optimal weight vector for (4) can be obtained as follows:

$\boldsymbol{w}_{0}=\boldsymbol{R}_{x}^{-1} \boldsymbol{G}\left(\boldsymbol{G}^{\mathrm{H}} \mathrm{R}_{x}^{-1} \boldsymbol{G}\right)^{-1} \boldsymbol{c}$.

It is shown by simulations that the proposed adaptive array beamformer with $\mathrm{MBC}$ demonstrates the capability of receiving multiple signals as well as suppressing incoherent jammers.

However, the effectiveness of the above proposed adaptive beamformer will be deteriorated when there exists coherence between a desired signal and jammers. This is due to the fact that the eigenstructure of the correlation matrix $\boldsymbol{R}_{x}$ will be destroyed because the rank of $\boldsymbol{R}_{x}$ is affected by the coherence between the desired signals and jammers.

\section{An iterative matrix reconstruction scheme}

To deal with the coherent jamming problem, it is appropriate to consider a possible manner for restoring the desired eigenstructure of $\boldsymbol{R}_{x}$. It is well known in the literature that the spatial smoothing (SS) algorithm developed by [6] is effective in curing the coherent problem for bearing estimation. The Toeplitz approximation approach of [3] is originally developed for bearing estimation in the coherent source environment. According to the SS algorithm, we can modify the expression of the optimal weight vector by replacing $\boldsymbol{R}_{x}$ in (5) with the spatial smoothed correlation matrix $\overline{\boldsymbol{R}}_{x}$ which is given by $\overline{\boldsymbol{R}}_{x}=(1 / J) \sum_{j=1}^{J} \boldsymbol{R}_{x}^{j}$, where $\boldsymbol{R}_{x}^{j}$ denotes the ensemble correlation matrix of the data vector received by the $j$ th subarray from the partition of the original array. However, the resolution capability of the array is reduced due to the effective aperture size decreases as the size of the subarrays decreases. Therefore, we resort to the following matrix reconstruction scheme without sacrificing the effective aperture size of the original array. After computing the correlation matrix $\boldsymbol{R}_{x}$ from (3), a reconstructed $M \times M$ matrix is given by

$\hat{\boldsymbol{R}}_{x}=\left[\hat{R}_{i j}\right]=[\hat{R}(i-j)]$, where

$\hat{R}(-m)=\frac{1}{M-m} \sum_{i=1}^{M-m} R_{i(i+m)}, \quad 0 \leqslant m<M$,

$\hat{R}(m)=\hat{R}^{*}(-m)$,

where the superscript $*$ represents conjugate operation. Eq. (7) reveals that the resulting matrix $\hat{\boldsymbol{R}}_{x}$ is also Hermitian with size $M \times M$. In fact, the reconstruction scheme is similar to the original Toeplitz approximation approach of [3].

In general, the reconstructed matrix $\hat{\boldsymbol{R}}_{x}$ would not have the desired eigenstructure property that its minimum eigenvalue has a multiplicity of $(M-K)$ unless the array size $M$ is infinite. Therefore, we propose an iterative algorithm to make the reconstructed matrix possess both the ToeplitzHermitian and the desired eigenstructure properties. First, the problem of reconstructing the desired eigenstructure from the estimated Toeplitz matrix $\hat{\boldsymbol{R}}_{x}$ is solved by performing the following minimization problem:

$\underset{\tilde{\boldsymbol{R}}_{x \in S_{E}}}{\operatorname{minimize}}\left|\tilde{\boldsymbol{R}}_{x}-\hat{\boldsymbol{R}}_{x}\right|$,

where $S_{E}$ denotes the set of matrices that their $(M-K)$ smallest eigenvalues are positive and equal. The notation $|\boldsymbol{Q}|$ used in (8) represents $|\boldsymbol{Q}|=\left(\sum_{i=1}^{M} \sum_{j=1}^{M}\left|q_{i j}\right|^{2}\right)^{1 / 2}$ with the $M \times M$ matrix $\boldsymbol{Q}=\left[q_{i j}\right]$. The optimal solution for (8), denoted as $\widetilde{\boldsymbol{R}}_{x o}$, in minimum metric distance sense is given by [7]

$\tilde{\boldsymbol{R}}_{x o}=\sum_{k=1}^{K} \lambda_{k} \boldsymbol{e}_{k} \boldsymbol{e}_{k}^{\mathrm{H}}+\lambda_{\mathrm{av}} \sum_{k=K+1}^{M} \boldsymbol{e}_{k} \boldsymbol{e}_{k}^{\mathrm{H}}$,

where $\lambda_{1} \geqslant \lambda_{2} \geqslant \cdots \lambda_{M}$ and $\boldsymbol{e}_{m}, m=1,2, \ldots, M$ are the eigenvalues and the corresponding eigenvectors of $\hat{\boldsymbol{R}}_{x}$, respectively, and $\lambda_{\mathrm{av}}$ is the average of $\lambda_{P+1}, \lambda_{P+2}, \ldots, \lambda_{M}$. In practice, it is generally the case that the total number $K$ of signal sources is unknown for adaptive beamforming. Hence, we resort to a suboptimal solution given by

$\tilde{\boldsymbol{R}}_{x s}=\sum_{k=1}^{P} \lambda_{k} \boldsymbol{e}_{k} \boldsymbol{e}_{k}^{\mathrm{H}}+\lambda_{\mathrm{av}} \sum_{k=P+1}^{M} \boldsymbol{e}_{k} \boldsymbol{e}_{k}^{\mathrm{H}}$

for (8). Moreover, we note that the non-linear operations performed by (9) and (10) cannot guarantee to produce a Toeplitz matrix. On the other 
hand, the above matrix reconstruction scheme for obtaining $\hat{\boldsymbol{R}}_{x}$ from $\boldsymbol{R}_{x}$ may alter the eigenstructure of a matrix. Therefore, it cannot be ensured that the reconstructed matrix $\tilde{\boldsymbol{R}}_{x s}$ possesses both the Toeplitz-Hermitian and the desired eigenstructure properties. However, the goal can be achieved using an iterative algorithm in which operations for obtaining $\hat{\boldsymbol{R}}_{x}$ and $\widetilde{\boldsymbol{R}}_{x s}$ are performed alternatively. Consequently, we summarize the proposed iterative matrix reconstruction scheme step-by-step as follows:

Step 1 . Estimate $\boldsymbol{R}_{x}$ from the received signals. Then let the iteration number $i=0$ and $\widetilde{\boldsymbol{R}}_{x s}^{(0)}=\boldsymbol{R}_{x}$.

Step 2. Compute the matrix $\hat{\boldsymbol{R}}_{x}^{(i+1)}$ from $\widetilde{\boldsymbol{R}}_{x s}^{(i)}$ by using the operation of (6).

Step 3. Compute the matrix $\widetilde{\boldsymbol{R}}_{x s}^{(i+1)}$ from $\hat{\boldsymbol{R}}_{x}^{(i+1)}$ by using the operation of (10).

Step 4. If the matrix norm $\left|\widetilde{R}_{x s}^{(i+1)}-\hat{\boldsymbol{R}}_{x}^{(i+1)}\right|>\varepsilon$, where $\varepsilon$ is a preset positive real number, then let $i=i+1$ and go to Step 2. Otherwise, go to the next step.

Step 5. Use the resulting $\widetilde{\boldsymbol{R}}_{x s}^{(i+1)}$ to replace $\boldsymbol{R}_{x}$ in obtaining the optimal weight vector given by (5).

Finally, the proof regarding the convergence of the proposed iterative scheme is presented in the appendix.

\section{Simulation results}

In this section, several simulation examples performed on a PC with Pentium-II CPU using MATLAB programming language are presented for illustration and comparison. For all simulation examples, we use a ULA with 10 array sensors and the interelement spacing equal to the half of the minimum wavelength of the signals with specified gain/null requirements. These are three signals impinging on the array from $-30^{\circ}, 0^{\circ}$, and $30^{\circ}$, respectively, off array broadside. Moreover, the first two signals are assumed to be the desired signals with gains all equal to one and the third signal is the jammer which is coherent with the first desired signal. All simulation results presented are obtained by averaging 50 independent runs with independent noise samples for each run. The value of $\varepsilon$ for terminating the iterative process is set to $10^{-6}$. In practice, the ensemble correlation matrix
$\boldsymbol{R}_{x}$ is not available. We resort to using the finite sample-size estimate $\overline{\boldsymbol{R}}_{x}$ (or called the sample correlation matrix) to replace $\boldsymbol{R}_{x}$ and performing the iterative scheme proposed in Section 3 on $\overline{\boldsymbol{R}}_{x}$ instead of $\boldsymbol{R}_{x}$ for simulations. 1200 data snapshots are used for computing the necessary sample correlation matrices related to the ensemble correlation matrices. For each example, the beam pattern shown by dash curve represents the result based on the optimal weight vector given by (5).

Example 1. Here, the three signals have signal-tonoise power ratio (SNR) all equal to $0 \mathrm{~dB}$. The constraint vector $c$ is set to $[11]^{\mathrm{T}}$. Hence, $P$ is set to 2. Fig. 1 shows the beam patterns with and without using the proposed technique. Clearly, the proposed technique can cope with the problem of coherent jamming situation. The result of using the $\mathrm{SS}$ algorithm instead of the proposed technique is also plotted in Fig. 1 for comparison. For performing the SS algorithm, three subarrays with 8 sensors for each are used. Although the SS algorithm is able to alleviate the effect of coherent jammers, the proposed technique shows the advantages of deeper null in the coherent jammer direction and narrower beam widths over the SS algorithm.

Example 2. Here, the simulations of Example 1 are repeated except that the three signals have SNR equal to 0,10 , and $20 \mathrm{~dB}$, respectively. The resulting

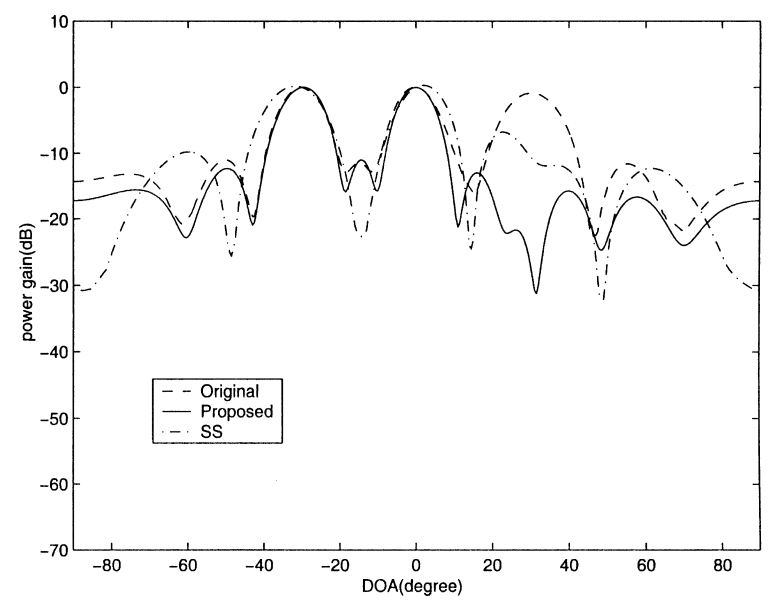

Fig. 1. The beampatterns for Example 1. 
beam patterns with and without using the proposed technique are shown in Fig. 2, while the result of using the SS algorithm is also depicted in Fig. 2. From Fig. 2, we observe again that the proposed technique outperforms the SS algorithm.

Example 3. In this example, we repeat the simulations of Example 1 except that the constraint vector $c$ is set to $\left[\begin{array}{lll}1 & 1 & 0\end{array}\right]^{\mathrm{T}}$. Thus, $P$ is equal to 3 in this case. Fig. 3 shows that the resulting beam patterns with and without using the proposed technique, and the result of using the SS algorithm. Although the two techniques provide very deep null in the coherent

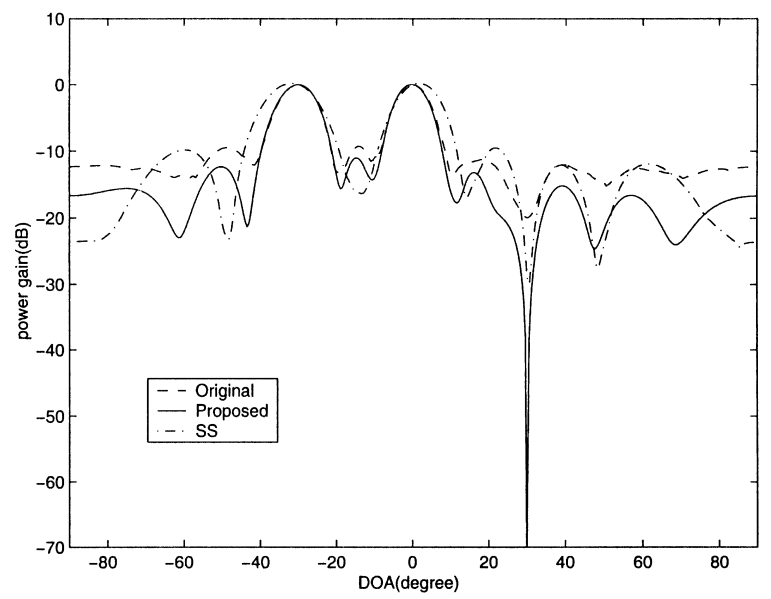

Fig. 2. The beampatterns for Example 2.

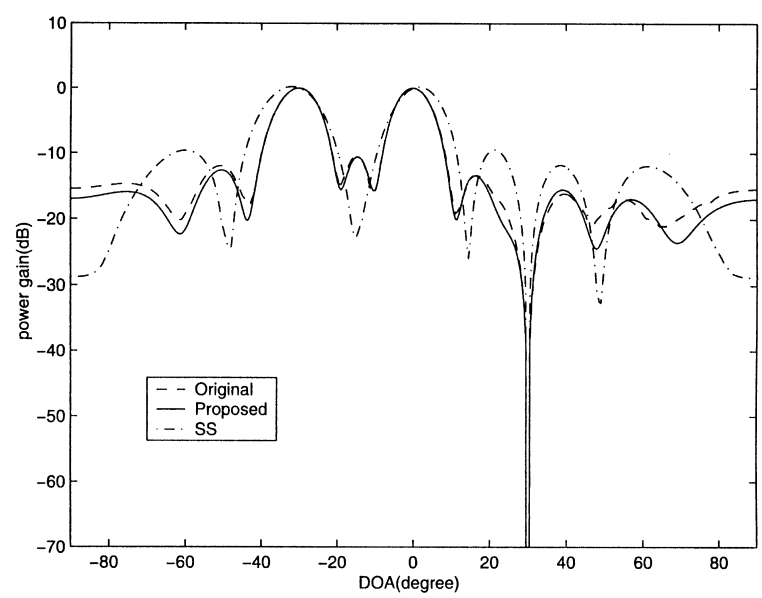

Fig. 3. The beampatterns for Example 3. jammer direction, the result of using proposed technique possesses narrower beam widths and lower sidelobes than those of using the SS algorithm.

\section{Conclusion}

This paper has presented a technique for adaptive beamforming using a uniform linear array with multiple signal gain/null specifications in addition to jammer suppression. An iterative matrix reconstruction scheme has further been proposed to incorporate the technique for dealing with the situation of coherent jammers. The convergence property of the proposed iterative scheme has also been analyzed. Simulation results have shown that the proposed technique can effectively cure the problem of adaptive beamforming with multiplebeam constraints in the presence of coherent jammers.

\section{Appendix A}

Here, we prove the convergence of the iterative scheme proposed in Section 3. Given an arbitrary matrix as the initial point, denoted as $\widetilde{\boldsymbol{R}}_{x s}^{0}$, the proposed iterative scheme generates a matrix sequence $\quad S_{R}=\left\{\widetilde{\boldsymbol{R}}_{x s}^{0}, \hat{\boldsymbol{R}}_{x}^{(1)}, \widetilde{\boldsymbol{R}}_{x s}^{(1)}, \hat{\boldsymbol{R}}_{x}^{(2)}, \ldots, \hat{\boldsymbol{R}}_{x}^{(i)}, \widetilde{\boldsymbol{R}}_{x s}^{(i)}\right.$, $\left.\hat{\boldsymbol{R}}_{x}^{(i+1)}, \widetilde{\boldsymbol{R}}_{x s}^{(i+1)}, \ldots\right\}$ by the following recursive manner: First, obtain $\hat{\boldsymbol{R}}_{x}^{(i+1)}$ from $\widetilde{\boldsymbol{R}}_{x s}^{(i)}$ by using the operation shown by (6). Second, obtain $\tilde{\boldsymbol{R}}_{x s}^{(i+1)}$ from $\hat{\boldsymbol{R}}_{x}^{(i+1)}$ by using the operation shown by (10), for $i=0,1,2, \ldots$. Moreover, we observe that the operations shown by (7) and (10) are norm-reduced and constant trace operations because

$$
|\hat{R}(-m)| \leqslant \frac{1}{M-m} \sum_{i=1}^{M-m}\left|R_{i(i+m)}\right|, \quad 0 \leqslant m<M,
$$

hence

$$
\left.\hat{R}(-m)\right|^{2} \leqslant \frac{1}{M-m} \sum_{i=1}^{M-m}\left|R_{i(i+m)}\right|^{2}, \quad 0 \leqslant m<M
$$


and

$\operatorname{trace}\left[\hat{\boldsymbol{R}}_{x}\right]=M \hat{R}(0)=\sum_{i=1}^{M} R_{i j}=\operatorname{trace}\left[\boldsymbol{R}_{x}\right]$,

and

$\left|\tilde{\boldsymbol{R}}_{x s}\right|^{2}=\sum_{k=1}^{P} \lambda_{k}^{2}+(M-P) \lambda_{\mathrm{av}}^{2} \leqslant \sum_{k=1}^{M} \lambda_{k}^{2}=\left|\boldsymbol{R}_{x}\right|^{2}$,

and

$$
\begin{aligned}
\operatorname{trace}\left[\tilde{\boldsymbol{R}}_{x s}\right] & =\sum_{k=1}^{P} \lambda_{k}+(M-P) \lambda_{\mathrm{av}} \\
& =\sum_{k=1}^{M} \lambda_{k}=\operatorname{trace}\left[\boldsymbol{R}_{x}\right] .
\end{aligned}
$$

Since $\hat{\boldsymbol{R}}_{x s}^{(i+1)}$ is the optimal solution of (6) when $\boldsymbol{R}_{x}=\widetilde{\boldsymbol{R}}_{x s}^{(i)}$, we have

$\left|\hat{\boldsymbol{R}}_{x}^{(i)}-\widetilde{\boldsymbol{R}}_{x s}^{(i)}\right| \geqslant\left|\hat{\boldsymbol{R}}_{x}^{(i+1)}-\tilde{\boldsymbol{R}}_{x s}^{(i)}\right|$.

Similarly, $\widetilde{\boldsymbol{R}}_{x s}^{(i+1)}$ is obtained by the norm-reduced operation of (10) when $\boldsymbol{R}_{x}=\hat{\boldsymbol{R}}_{x}^{(i+1)}$, we have

$\left|\hat{\boldsymbol{R}}_{x}^{(i+1)}-\widetilde{\boldsymbol{R}}_{x s}^{(i)}\right| \geqslant\left|\hat{\boldsymbol{R}}_{x}^{(i+1)}-\widetilde{\boldsymbol{R}}_{x s}^{(i+1)}\right|$.

From (A.6) and (A.7), it follows that

$\left|\hat{\boldsymbol{R}}_{x}^{(i)}-\widetilde{\boldsymbol{R}}_{x s}^{(i)}\right| \geqslant\left|\hat{\boldsymbol{R}}_{x}^{(i+1)}-\tilde{\boldsymbol{R}}_{x s}^{(i+1)}\right|$.

Next, define a real non-negative sequence $\left\{d_{i}\right\}$ as

$d_{i}=\left|\hat{\boldsymbol{R}}_{x}^{(i)}-\widetilde{\boldsymbol{R}}_{x s}^{(i)}\right|$

with $i=1,2, \ldots$. From (A.8) and (A.9), we note that the descending sequence $\left\{d_{i}\right\}$ must converge to some non-negative constant $c$ [5]. If $c=0$, then $\hat{\boldsymbol{R}}_{x}^{(i)}=\widetilde{\boldsymbol{R}}_{x s}^{(i)}$ as $i$ approaches $\infty$. This leads to the result that the matrix sequence $S_{R}$ converges. On the other hand, if $c>0$, then we have from (A.6)-(A.9) that

$\left|\hat{\boldsymbol{R}}_{x}^{(i)}-\widetilde{\boldsymbol{R}}_{x s}^{(i)}\right|=\left|\hat{\boldsymbol{R}}_{x}^{(i+1)}-\widetilde{\boldsymbol{R}}_{x s}^{(i)}\right|=\left|\hat{\boldsymbol{R}}_{x}^{(i+1)}-\widetilde{\boldsymbol{R}}_{x s}^{(i+1)}\right|$

as $i$ approaches $\infty$. It follows from (A.10) that $\hat{\boldsymbol{R}}^{(i)}$ and $\hat{\boldsymbol{R}}^{(i+1)}$ are the solution of (5) when $\boldsymbol{R}_{x}=\widetilde{\boldsymbol{R}}_{x s}^{(i)}$. Hence, $\hat{\boldsymbol{R}}_{x}^{(i)}=\hat{\boldsymbol{R}}_{x}^{(i+1)}$ since the solution for the minimization problem of (5) is unique. Therefore, the matrix subsequence $\left\{\hat{\boldsymbol{R}}_{x}^{(i)}\right\}$ converges. Similarly, the matrix subsequence $\left\{\tilde{\boldsymbol{R}}_{x s}^{(i)}\right\}$ also converges because $\widetilde{\boldsymbol{R}}_{x s}^{(i)}$ is obtained from (10) when $\boldsymbol{R}_{x}=\hat{\boldsymbol{R}}_{x}^{(i)}=\hat{\boldsymbol{R}}_{x}^{(i+1)}$ and, hence, $\widetilde{\boldsymbol{R}}_{x s}^{(i)}=\widetilde{\boldsymbol{R}}_{x s}^{(i+1)}$. As a result, we would expect that the two subsequences converge to two different matrices since $c>0$. Moreover, based on the facts that $\widetilde{\boldsymbol{R}}_{x s}^{(i)} \neq \hat{\boldsymbol{R}}_{x}^{(i+1)}$ and both the operations for obtaining $\hat{\boldsymbol{R}}_{x}$ and $\widetilde{\boldsymbol{R}}_{x s}$ are norm-reduced operations, we have

$\left|\widetilde{\boldsymbol{R}}_{x s}^{(i)}\right|>\left|\hat{\boldsymbol{R}}_{x}^{(i+1)}\right|>\left|\widetilde{\boldsymbol{R}}_{x s}^{(i+1)}\right|$.

This leads to the result that $\left|\tilde{\boldsymbol{R}}_{x s}^{(i)}\right|>\left|\tilde{\boldsymbol{R}}_{x s}^{(i+1)}\right|$. However, this contradicts the results $\hat{\boldsymbol{R}}_{x}^{(i)}=\hat{\boldsymbol{R}}_{x}^{(i+1)}$ obtained from (A.10). Consequently, $c$ must be zero. Both the operations for obtaining $\hat{\boldsymbol{R}}_{x}$ and $\tilde{\boldsymbol{R}}_{x s}$ are also constant trace operations, we can thus find that $\left|\boldsymbol{R}_{S}\right|>0$ for any $\boldsymbol{R}_{S} \in S_{R}$. Therefore, the proposed iterative matrix reconstruction scheme will not converge to the trivial solution. i.e., the null matrix. This completes the necessary proof.

\section{References}

[1] R.T. Compton Jr., Adaptive Antennas: Concepts and Performance, Prentice-Hall, Englewood Cliffs, New York, 1988, pp. 361-376.

[2] O.L. Frost, An algorithm for constrained adaptive array processing, Proc. IEEE 60 (August 1972) 926-935.

[3] S.Y. Kung, C.K. Lo, R. Foka, A Toeplitz approximation approach to coherent source direction finding, Proceedings of the ICASSP, Tokyo, Japan, April 1986, pp. 193-196.

[4] J.T. Mayhan, Area coverage adaptive nulling from geosynchronous satellites: phased arrays versus multiple-beam antennas, IEEE Trans. Antennas Propag. AP-34 (March 1986) 410-419.

[5] M.H. Protter, C.B. Morrey, A First Course in Real Analysis, Springer, New York, 1977.

[6] T.J. Shan, M. Wax, T. Kailath, On spatial smoothing for direction-of-arrival estimation of coherent signals, IEEE Trans. Acoust. Speech Signal Process. ASSP-33 (August 1985) 806-811.

[7] M. Wax, T. Kailath, Detection of signals by information theoretic criteria, IEEE Trans. Acoust. Speech Signal Process. ASSP-33 (April 1985) 387-392.

[8] K.-B. Yu, Adaptive beamforming for satellite communication with selective earth coverage and jammer nulling capability, IEEE Trans. Signal Process. 44 (December 1996) 3162-3166. 